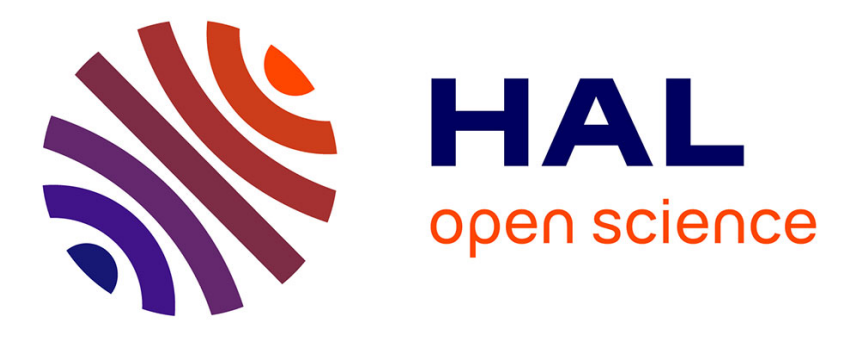

\title{
MIMO radar in the presence of modeling errors: A Cramér-Rao bound investigation
}

\author{
Duy Tran Nguyen, Alexandre Renaux, Remy Boyer, Sylvie Marcos, Pascal \\ Larzabal
}

\section{- To cite this version:}

Duy Tran Nguyen, Alexandre Renaux, Remy Boyer, Sylvie Marcos, Pascal Larzabal. MIMO radar in the presence of modeling errors: A Cramér-Rao bound investigation. ICASSP 2011 - 36th IEEE International Conference on Acoustics, Speech and Signal Processing, May 2011, Prague, Czech Republic. pp.2780 - 2783, 10.1109/ICASSP.2011.5947061 . hal-00771365

\section{HAL Id: hal-00771365}

https://hal-centralesupelec.archives-ouvertes.fr/hal-00771365

Submitted on 15 Mar 2020

HAL is a multi-disciplinary open access archive for the deposit and dissemination of scientific research documents, whether they are published or not. The documents may come from teaching and research institutions in France or abroad, or from public or private research centers.
L'archive ouverte pluridisciplinaire $\mathbf{H A L}$, est destinée au dépôt et à la diffusion de documents scientifiques de niveau recherche, publiés ou non, émanant des établissements d'enseignement et de recherche français ou étrangers, des laboratoires publics ou privés. 


\title{
MIMO RADAR IN THE PRESENCE OF MODELING ERRORS: A CRAMÉR-RAO BOUND INVESTIGATION
}

\author{
Nguyen Duy TRAN ${ }^{(1,2)}$, Alexandre RENAUX ${ }^{(1)}$, Rémy BOYER $^{(1)}$, Sylvie MARCOS ${ }^{(1)}$ and Pascal LARZABAL ${ }^{(2)}$ \\ (1) Université Paris-Sud 11 / CNRS / SUPELEC \\ Laboratoire des Signaux et Systèmes, \\ Supelec, 3, rue Joliot-Curie, 91192 Gif-sur-Yvette, France \\ ${ }^{(2)}$ Ecole Normale Supérieure de Cachan \\ Laboratoire SATIE / CNRS \\ 61 av. du Président Wilson, 94235 Cachan, France
}

\begin{abstract}
In this paper, we study the impact of modeling error on the receiver of a MIMO radar. Following other works on classical array processing, we derive closed-form expressions of the Cramér-Rao bounds for an observation model of a widely spaced MIMO radar affected by modeling error. We show that, as the signal-to-noise ratio increase, the Cramér-Rao bound and the mean square error of the maximum likelihood estimator of the angle-of-arrival do not fall into zero (contrary to the classical case without error modeling) and converge to a fixed limit for which we give a closed-form expression. Moreover, we give a simple closed-form expression of the critical value of the signal-to-noise ratio where this limitation of performance appear.
\end{abstract}

Index Terms- MIMO radar, modeling error.

\section{INTRODUCTION}

A Multi-Input Multi-Output (MIMO) radar is a system that uses simultaneously multiple antennas which both transmit and receive a set of probing waveforms to collect the targets information. The probing waveforms can be fully uncorrelated or partially correlated. The conventional phased array radar is a case of MIMO radar where all the transmitted waveforms are correlated. It is now known that a MIMO radar system has many advantages in comparison with a phased-array radar in terms of detection/estimation (see, e.g. [1] and [2]).

However such good performance can be achieved only when the observation model of the MIMO radar is well known because the powerful detection/estimation techniques used are often based on statistically optimal algorithms such as the maximum likelihood technique. Indeed, in the literature, the system model are supposed to be correct, i.e. the array response is supposed to be precisely known. However, in practice, the assumed model is different from the true one due to the variation of array element positions or the differences in element patterns. Even though this mismatch is usually regulated by a calibration procedure, the imperfections in the array still exist and degrade the system performance. Besides, a precious calibration procedure is expensive and time consuming. Hence, it is important to investigate the modeling errors in radar system to reduce the requirements without a noticeable degradation in the performance. This is why, in this paper, we focus on the influence of

This project was funded by both région Île-de-France and DIGITEO Research Park. modeling error at the receiver of a MIMO radar on the localization of a target. We consider for this analysis the so-called widely separated MIMO radar with linear arrays (possibly non-uniform or lacunar) where the localization parameter of the target are the angle-ofdeparture denoted and the angle-of-arrival. In the literature, there is few result for the behavior of MIMO radar in the presence of modeling errors. Mainly, an analysis via performance bounds of MIMO radar affected by modeling errors can be found in [3], [4], [5] and [6]. However, in these work only phase synchronization error are studied. Also, some works have been done in passive array processing. One can cite [7], [8] and recently [9]. Our approach follows the idea presented in [9] where the error is modeled by a Gaussian random vector added to the true steering vector.

In this work, we derive the Cramér-Rao bound w.r.t unknown target parameters in the context of MIMO radar in which the receiver suffers from an array response error. Then, the proposed closedform expressions are analyzed and it is shown that the Cramér-Rao bound and the mean square error of the maximum likelihood estimator of the angle-of-arrival do not fall into zero (contrary to the classical case without modeling error) and converge to a fixed limit for which we give a closed-form expression. Moreover, we give a simple closed-form expression of the critical value of the signal-tonoise ratio where this limitation of performance appear.

\section{PROBLEM SETUP}

We consider a MIMO radar equipped with well separated transmitting and receiving arrays. Both arrays are assumed to be centralsymmetric linear arrays where the numbers of transmit and receive antennas are denoted $M$ and $N$, respectively. In this context, a point target is located by two parameters: the angle-of-departure denoted by $\theta_{D}$ and the angle-of-arrival denoted by $\theta_{A}$. Consequently, the observation model is given by:

$$
\mathbf{y}(t)=\beta \mathbf{b}\left(\theta_{A}\right) \mathbf{a}^{T}\left(\theta_{D}\right) \mathbf{x}(t)+\mathbf{n}(t), t=1 \ldots T,
$$

where $T$ is the number of snapshots. The complex quantity $\beta$ is the target complex amplitude related to the RCS of the target. The vector $\mathbf{x}(t)$ contains each transmitted waveforms. We assume that these waveforms are orthogonal and have the following empirical covariance matrix:

$$
\mathbf{R}_{x}=\frac{1}{T} \sum_{t=1}^{T} \mathbf{x}(t) \mathbf{x}^{H}(t)=\sigma_{x}^{2} \mathbf{I}_{M} .
$$


The steering vectors have the following structures

$$
\begin{aligned}
& \mathbf{a}\left(\theta_{D}\right)= \\
& {\left[\exp \left(-j \frac{2 \pi}{\lambda} a_{1} \sin \theta_{D}\right), \ldots, \exp \left(-j \frac{2 \pi}{\lambda} a_{M} \sin \theta_{D}\right)\right]^{T}}
\end{aligned}
$$

and

$$
\begin{aligned}
& \mathbf{b}\left(\theta_{A}\right)= \\
& {\left[\exp \left(-j \frac{2 \pi}{\lambda} b_{1} \sin \theta_{A}\right), \ldots, \exp \left(-j \frac{2 \pi}{\lambda} b_{N} \sin \theta_{A}\right)\right]^{T},}
\end{aligned}
$$

where $\lambda$ is the wavelength, where the quantities $a_{i}, i=1 \ldots M$, and $b_{j}, j=1 \ldots N$, are the nominal sensor positions (w.r.t. a reference point) of transmitting and receiving arrays, respectively. Finally, the noise vectors $\{\mathbf{n}(t)\}_{t=1}^{T}$ are assumed to be independent and identically distributed circularly symmetric complex Gaussian with zero-mean and covariance matrix $\mathbf{R}_{n}=\sigma_{n}^{2} \mathbf{I}_{N}$.

In this paper, we consider that the true steering vector of the receiving array, $\mathbf{b}_{\text {true }}\left(\theta_{A}\right)$, is subject to a modeling error represented by an additive random vector $\mathbf{e}$. In other words,

$$
\mathbf{b}\left(\theta_{A}\right)=\mathbf{b}_{\text {true }}\left(\theta_{A}\right)+\mathbf{e}
$$

where $\mathbf{e}$ is assumed to be jointly circular and Gaussian distributed, namely $\mathbf{e} \sim \mathcal{C N}\left(0, \sigma_{e}^{2} \mathbf{I}_{N}\right)$. Moreover, $\mathbf{e}$ is assumed to be statistically independent of the noise vector $\mathbf{n}(t), \forall t$. Consequently, the complex data vector received by such a MIMO radar can be written as

$$
\mathbf{y}(t)=\beta \mathbf{b}_{\text {true }}\left(\theta_{A}\right) \mathbf{a}^{T}\left(\theta_{D}\right) \mathbf{x}(t)+\beta \mathbf{e a}^{T}\left(\theta_{D}\right) \mathbf{x}(t)+\mathbf{n}(t) .
$$

The unknown parameters vector is $\boldsymbol{\Theta}=\left[\theta_{D}, \theta_{A}, \beta_{R}, \beta_{I}\right]^{T}$ where $\beta_{R}$ and $\beta_{I}$ denote the real part of $\beta$ and the imaginary part of $\beta$, respectively.

Finally, note that ${ }^{1}$ in this case the likelihood of the observations is complex circular Gaussian distributed with both parameterized mean and covariance matrix, i.e., by letting $\mathbf{y}=\left[\mathbf{y}^{T}(1) \ldots \mathbf{y}^{T}(T)\right]^{T} \in$ $\mathbb{C}^{N T}, \mathbf{y} \mid \boldsymbol{\Theta} \sim \mathcal{C} \mathcal{N}(\mathbf{m}(\Theta), \mathbf{R}(\Theta))$ where

$$
\mathbf{m}(\Theta)=
$$$$
\operatorname{vec}\left(\left[\beta \mathbf{b}_{\text {true }}\left(\theta_{A}\right) \mathbf{a}^{T}\left(\theta_{D}\right) \mathbf{x}(1) \ldots \beta \mathbf{b}_{\text {true }}\left(\theta_{A}\right) \mathbf{a}^{T}\left(\theta_{D}\right) \mathbf{x}(T)\right]\right),
$$

where vec(.) denotes the vec operator and

$$
\mathbf{R}(\boldsymbol{\Theta})=|\beta|^{2} \sigma_{e}^{2} \mathbf{s}\left(\theta_{D}\right) \mathbf{s}^{H}\left(\theta_{D}\right) \otimes \mathbf{I}_{N}+\sigma_{n}^{2} \mathbf{I}_{N T}
$$

where

$$
\mathbf{s}\left(\theta_{D}\right)=\left[\mathbf{a}^{T}\left(\theta_{D}\right) \mathbf{x}(1) \ldots \mathbf{a}^{T}\left(\theta_{D}\right) \mathbf{x}(T)\right]^{T} .
$$

We note that the full parameter vector parameterizes the mean while only $\theta_{D}, \beta_{R}$, and $\beta_{I}$ parameterize the covariance matrix of the observations.

\footnotetext{
${ }^{1}$ This is quite different from the classical case (in the MIMO radar context or in the array processing context) where only the mean or the covariance matrix are parameterized. Note also that the analysis of modeling error on both transmitter and receiver seems to be a very complex work. Indeed, in this case the observations vector is the sum of a deterministic vector plus a Gaussian vector plus the product of two Gaussian vector depend on the first Gaussian vector.
}

\section{CRAMÉR-RAO BOUND}

For a general Gaussian parameterized model such that

$$
\mathbf{y} \mid \Theta \sim \mathcal{C N}(\mathbf{m}(\Theta), \mathbf{R}(\Theta))
$$

its is well known that the element $(i, j)$ of the Fisher information matrix $\mathbf{F}(\Theta)$ is given by the Slepian-Bang formula (see, e.g., [10])

$$
\begin{aligned}
&\{\mathbf{F}(\boldsymbol{\Theta})\}_{i, j}= \operatorname{Tr}\left\{\mathbf{R}^{-1}(\boldsymbol{\Theta}) \frac{\partial \mathbf{R}(\boldsymbol{\Theta})}{\partial\{\boldsymbol{\Theta}\}_{i}} \mathbf{R}^{-1}(\boldsymbol{\Theta}) \frac{\partial \mathbf{R}(\boldsymbol{\Theta})}{\partial\{\boldsymbol{\Theta}\}_{j}}\right\} \\
&+2 \operatorname{Re}\left\{\frac{\partial \mathbf{m}^{H}(\boldsymbol{\Theta})}{\partial\{\boldsymbol{\Theta}\}_{i}} \mathbf{R}^{-1}(\boldsymbol{\Theta}) \frac{\partial \mathbf{m}(\boldsymbol{\Theta})}{\partial\{\boldsymbol{\Theta}\}_{j}}\right\},(10)
\end{aligned}
$$

where $\{\boldsymbol{\Theta}\}_{i=1, \ldots, 4}$ denote the $i^{\text {th }}$ element of the vector $\boldsymbol{\Theta}$. Without loss of generality, the reference points for the transmitting and receiving arrays are choosing such that

$$
\mathbf{a}^{H}\left(\theta_{D}\right) \dot{\mathbf{a}}\left(\theta_{D}\right)=0 \text { and } \mathbf{b}_{\text {true }}^{H}\left(\theta_{A}\right) \dot{\mathbf{b}}_{\text {true }}\left(\theta_{A}\right)=0,
$$

where we define $\dot{\mathbf{a}}\left(\theta_{D}\right)=\frac{\partial \mathbf{a}\left(\theta_{D}\right)}{\partial \theta_{D}}$, and $\dot{\mathbf{b}}_{\text {true }}\left(\theta_{A}\right)=\frac{\partial \mathbf{b}_{\text {true }}\left(\theta_{A}\right)}{\partial \theta_{A}}$. Let us set $\dot{\mathbf{s}}\left(\theta_{D}\right)=\left[\dot{\mathbf{a}}^{T}\left(\theta_{D}\right) \mathbf{x}(1) \ldots \dot{\mathbf{a}}^{T}\left(\theta_{D}\right) \mathbf{x}(T)\right]^{T}$, after calculus, one obtains

$$
\begin{gathered}
\mathbf{R}^{-1}(\boldsymbol{\Theta})= \\
\frac{1}{\sigma_{n}^{2}}\left(\mathbf{I}_{T}-\frac{|\beta|^{2} \sigma_{e}^{2}}{\sigma_{n}^{2}+|\beta|^{2} T M \sigma_{x}^{2} \sigma_{e}^{2}} \mathbf{s}\left(\theta_{D}\right) \mathbf{s}^{H}\left(\theta_{D}\right)\right) \otimes \mathbf{I}_{N} \\
\frac{\partial \mathbf{m}(\boldsymbol{\Theta})}{\partial \theta_{D}}=\operatorname{vec}\left(\left[\cdots \beta \mathbf{b}_{\text {true }}\left(\theta_{A}\right) \dot{\mathbf{a}}^{T}\left(\theta_{D}\right) \mathbf{x}(t) \cdots\right]\right) \\
\frac{\partial \mathbf{m}(\boldsymbol{\Theta})}{\partial \theta_{A}}=\operatorname{vec}\left(\left[\cdots \beta \dot{\mathbf{b}}_{\text {true }}\left(\theta_{A}\right) \mathbf{a}^{T}\left(\theta_{D}\right) \mathbf{x}(t) \cdots\right]\right) \\
\frac{\partial \mathbf{m}(\boldsymbol{\Theta})}{\partial \beta_{R}}=\operatorname{vec}\left(\left[\cdots \mathbf{b}_{t r u e}\left(\theta_{A}\right) \mathbf{a}^{T}\left(\theta_{D}\right) \mathbf{x}(t) \cdots\right]\right) \\
\frac{\partial \mathbf{m}(\boldsymbol{\Theta})}{\partial \beta_{I}}=j \frac{\partial \mathbf{m}(\boldsymbol{\Theta})}{\partial \beta_{R}} \\
\frac{\partial \theta_{D}(\boldsymbol{\Theta})}{\partial \theta_{D}}=|\beta|^{2} \sigma_{e}^{2}\left[\dot{\mathbf{s}}\left(\theta_{D}\right) \mathbf{s}^{H}\left(\theta_{D}\right)+\mathbf{s}\left(\theta_{D}\right) \dot{\mathbf{s}}^{H}\left(\theta_{D}\right)\right] \otimes \mathbf{I}_{N} \\
\frac{\partial \mathbf{R}(\boldsymbol{\Theta})}{\partial \beta_{R}}=2 \beta_{R} \sigma_{e}^{2} \mathbf{s}\left(\theta_{D}\right) \mathbf{s}^{H}\left(\theta_{D}\right) \otimes \mathbf{I}_{N} \\
\partial \theta_{A}
\end{gathered}
$$

and

$$
\frac{\partial \mathbf{R}(\boldsymbol{\Theta})}{\partial \beta_{I}}=2 \beta_{I} \sigma_{e}^{2} \mathbf{s}\left(\theta_{D}\right) \mathbf{s}^{H}\left(\theta_{D}\right) \otimes \mathbf{I}_{N} .
$$

Note also that

$$
\dot{\mathbf{a}}=\left[\cdots-j \frac{2 \pi}{\lambda} a_{k} \cos \left(\theta_{D}\right) \exp \left(-j \frac{2 \pi}{\lambda} a_{k} \sin \theta_{D}\right) \cdots\right]^{T},
$$

$k=1, \ldots, M$ and

$$
\dot{\mathbf{b}}=\left[\cdots-j \frac{2 \pi}{\lambda} b_{k} \cos \left(\theta_{A}\right) \exp \left(-j \frac{2 \pi}{\lambda} b_{k} \sin \theta_{A}\right) \cdots\right]^{T},
$$


$k=1, \ldots, N$. Plugging these results into Eqn. (10) and after tedious but straightforward calculus one obtains the elements of the Fisher information matrix

$$
\begin{aligned}
& \{\mathbf{F}(\Theta)\}_{1,1}=\frac{8 \pi^{2}|\beta|^{2} T N \sigma_{x}^{2}}{\lambda^{2} \sigma_{n}^{2}} \cos ^{2}\left(\theta_{D}\right) \sum_{k=1}^{M} a_{k}^{2} \\
& \times\left(1+\frac{|\beta|^{2} T M \sigma_{x}^{2} \sigma_{e}^{4}}{\sigma_{n}^{2}+|\beta|^{2} T M \sigma_{x}^{2} \sigma_{e}^{2}}\right), \\
& \{\mathbf{F}(\boldsymbol{\Theta})\}_{2,2}=\frac{\frac{8 \pi^{2}}{\lambda^{2}}|\beta|^{2} T M \sigma_{x}^{2}}{\sigma_{n}^{2}+|\beta|^{2} T M \sigma_{x}^{2} \sigma_{e}^{2}} \cos ^{2} \theta_{A} \sum_{k=1}^{N} b_{k}^{2}, \\
& \{\mathbf{F}(\boldsymbol{\Theta})\}_{3,3}=N\left(\frac{2 \beta_{R} T M \sigma_{x}^{2} \sigma_{e}^{2}}{\sigma_{n}^{2}+|\beta|^{2} T M \sigma_{x}^{2} \sigma_{e}^{2}}\right)^{2} \\
& +\frac{2 T N M \sigma_{x}^{2}}{\sigma_{n}^{2}+|\beta|^{2} T M \sigma_{x}^{2} \sigma_{e}^{2}} \\
& \{\mathbf{F}(\Theta)\}_{4,4}=N\left(\frac{2 \beta_{I} T M \sigma_{x}^{2} \sigma_{e}^{2}}{\sigma_{n}^{2}+|\beta|^{2} T M \sigma_{x}^{2} \sigma_{e}^{2}}\right)^{2} \\
& +\frac{2 T N M \sigma_{x}^{2}}{\sigma_{n}^{2}+|\beta|^{2} T M \sigma_{x}^{2} \sigma_{e}^{2}}, \\
& \begin{aligned}
\{\mathbf{F}(\boldsymbol{\Theta})\}_{3,4} & =\{\mathbf{F}(\boldsymbol{\Theta})\}_{4,3} \\
& =4 N \beta_{R} \beta_{I}\left(\frac{T M \sigma_{x}^{2} \sigma_{e}^{2}}{\sigma_{n}^{2}+|\beta|^{2} T M \sigma_{x}^{2} \sigma_{e}^{2}}\right)^{2},
\end{aligned}
\end{aligned}
$$

and all the other elements of the Fisher information matrix are equal to zero leading to a strong block diagonal structure (only $\beta_{R}$ and $\beta_{I}$ are coupled).

Consequently, the Cramér-Rao bounds (i.e., the diagonal elements of the Fisher information matrix inverse) are given by

$$
\begin{gathered}
\operatorname{CRB}\left(\theta_{D}\right)= \\
\frac{\lambda^{2} \sigma_{n}^{2}\left(\sigma_{n}^{2}+|\beta|^{2} T M \sigma_{x}^{2} \sigma_{e}^{2}\right)}{8 \pi^{2}|\beta|^{2} T N \sigma_{x}^{2}\left(\sigma_{n}^{2}+|\beta|^{2} T M \sigma_{x}^{2} \sigma_{e}^{2}\left(1+\sigma_{e}^{2}\right)\right) \cos ^{2}\left(\theta_{D}\right) \sum_{k=1}^{M} a_{k}^{2}},
\end{gathered}
$$

and

$$
C R B\left(\theta_{A}\right)=\frac{\sigma_{n}^{2}+|\beta|^{2} T M \sigma_{x}^{2} \sigma_{e}^{2}}{\frac{8 \pi^{2}}{\lambda^{2}}|\beta|^{2} T M \sigma_{x}^{2} \cos ^{2} \theta_{A} \sum_{k=1}^{N} b_{k}^{2}} .
$$

Due to the lack of space we do not give the closed-form expressions of $C R B\left(\beta_{R}\right)$ and $C R B\left(\beta_{I}\right)$, which are less interesting and which can easily be obtained from Eqn. (25), Eqn. (26) and Eqn. (27).

\section{SIMULATION RESULTS}

In order to analyze the Cramér-Rao bounds behavior, we perform in this Section some simulation results. The scenario is the following: the transmit array is a uniform linear array with $M=4$ sensors and with inter-element spacing (in unit of wavelengths) equals to 0.5 and the receive array is a uniform linear array with $N=4$ sensors and with inter-element spacing (in unit of wavelengths) is 0.5 . The orthogonal MIMO radar waveforms are generated using Hadamard codes with $T=32$ snapshots. We put the angle-of-departure and the angle-of-arrival of the target at $\theta_{D}=67.5^{\circ}$ and $\theta_{A}=22.5^{\circ}$ and we assume that $\beta=1+j$. The total transmitted power is $M \sigma_{x}^{2}=1$. Figure 1 shows the Cramér-Rao bounds for parameters of interest versus the Array Signal-to-Noise Ratio (ASNR) where we define $A S N R=\frac{M N \sigma_{x}^{2}}{\sigma_{n}^{2}}$. Note that we do not plot $C R B\left(\beta_{I}\right)$ since it has the same behavior as $C R B\left(\beta_{R}\right)$. For comparison, we also draw the performance of the maximum likelihood estimator (MLE) evaluated over 1000 Monte Carlo trials. This first simulation represents the behavior of the Cramér-Rao bounds and of the MLE without modeling error, i.e., with $\sigma_{e}^{2}=0$. We note that one observes the classical behavior of the Cramér-Rao bounds which decrease linearly when the ASNR increase. We also note that the MLE achieve asymptotically the Cramér-Rao bound (as $A S N R \rightarrow \infty$ ) [11] and we observe the classical threshold of the MSE of the MLE at low ASNR.

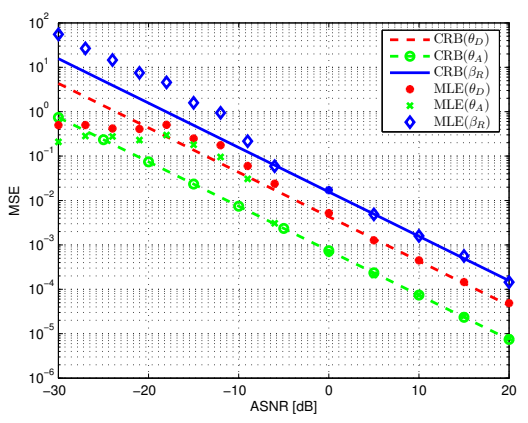

Fig. 1. Maximum Likelihood estimator empirical mean square error and Cramér-Rao bounds versus ASNR without modelling error

Figure 2 shows the behavior of the Cramér-Rao bounds and of the MLE with error modeling where $\sigma_{e}^{2}=0.1$.

Again, we observe the asymptotic efficiency of the MLE but, when $A N S R \rightarrow \infty$, the Cramér-Rao bound and the MSE of the MLE of the angle-of-arrival $\theta_{A}$ do not fall into zero and converge to a fixed limit that can be derived from Eqn. (29) and which is equal to

$$
\lim _{A S N R \rightarrow \infty} C R B\left(\theta_{A}\right)=\frac{\sigma_{e}^{2}}{\frac{8 \pi^{2}}{\lambda^{2}} \cos ^{2} \theta_{A} \sum_{k=1}^{N} b_{k}^{2}} .
$$

Note that this value is independent of $\beta$. This convergence means that for a given "power" level of modeling error $\sigma_{e}^{2}$, if the ASNR is over a certain threshold value, no improvement on the estimation 


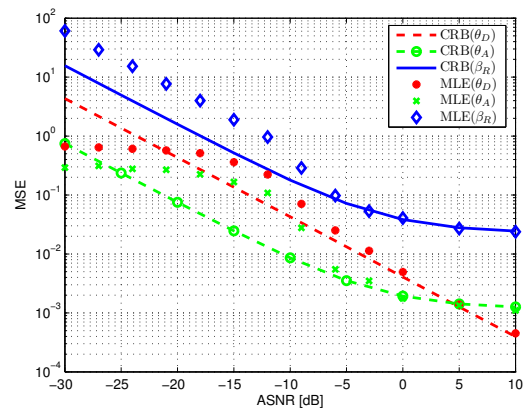

Fig. 2. Maximum Likelihood estimator empirical mean square error and Cramér-Rao bounds versus ASNR with $\sigma_{e}^{2}=0.1$.

performance can be done. Quantitatively, we calculate this threshold value of the ANSR denoted $A S N R_{\text {threshold }}$ as the value at which, $C R B\left(\theta_{A}\right)=(1+\epsilon) \times \lim _{A S N R \rightarrow \infty} C R B\left(\theta_{A}\right)$. We obtain the following simple closed-form expression

$$
A S N R_{\text {threshold }}=10 \log _{10} \frac{N}{\epsilon \sigma_{e}^{2} T|\beta|^{2}} .
$$

This expression shows that $A S N R_{\text {threshold }}$ is linear (in $\mathrm{dB}$ ) w.r.t. $\sigma_{e}^{2}$. Note that the same behavior occurs on $\beta_{R}$ and $\beta_{I}$, i.e., when $A N S R \rightarrow \infty$, the Cramér-Rao bound and the MSE of the MLE do not fall into zero and converge to a fixed limit which is given by

$$
\lim _{A S N R \rightarrow \infty} C R B\left(\beta_{R}\right)=\frac{\sigma_{e}^{2}\left(|\beta|^{2}+2 \sigma_{e}^{2} \beta_{I}\right)}{2 N\left(1+2 \sigma_{e}^{2}\right)},
$$

and

$$
\lim _{A S N R \rightarrow \infty} C R B\left(\beta_{I}\right)=\frac{\sigma_{e}^{2}\left(|\beta|^{2}+2 \sigma_{e}^{2} \beta_{R}\right)}{2 N\left(1+2 \sigma_{e}^{2}\right)} .
$$

Finally, we plotted on Figure 3 the Cramér-Rao bounds versus $\sigma_{e}^{2}$ with $A S N R=20 d B$. Again, we note that both $\theta_{A}$ and $\beta$ are affected by the modeling error while $\theta_{D}$ is not affected.

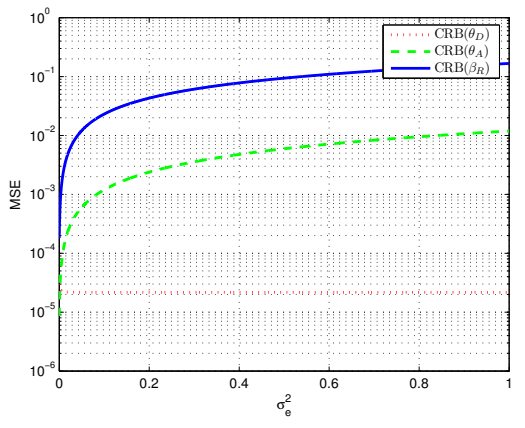

Fig. 3. Cramér-Rao bounds versus $\sigma_{e}^{2}$ with $A S N R=20 d B$

\section{CONCLUSION}

An investigation, by way of the Cramér-Rao bound, of the influence of modeling error at the receiver of a widely separated MIMO radar to localize a target was conducted. For that purpose, we have derived closed-form expressions of the Fisher information matrix and shown its block diagonal structure to deduce the Cramér-Rao bounds expressions of the angle-of-arrival and of the angle-of-departure. We have shown that, under a certain of signal-to-noise ratio, the performance of the system can not be improved. Finally, we have proposed a simple formula to evaluate this critical value of the signal-to-noise ratio.

\section{REFERENCES}

[1] J. Li and P. Stoica, MIMO Radar Signal Processing. New York: Wiley, 2009.

[2] E. Fishler, A. Haimovich, R. Blum, L. Cimini, D. Chizhik, and R. Valenzuela, "MIMO radar: an idea whose time has come," in Proc. of the IEEE Int. Conf. on Radar, Apr. 2004, pp. 71-78.

[3] H. Godrich, A. M. Haimovich, and H. V. Poor, "An analysis of phase synchronization mismatch sensitivity for coherent mimo radar systems," in Proc. Third International Workshop on Computational Advances in Multi-Sensor Adaptive Processing (CAMSAP), Dec. 2009. 
[4] Q. He and R. S. Blum, "Cramér-Rao bound for MIMO radar target localization with phase errors," IEEE Signal Processing Letters, no. 1, pp. 83-86, Jan. 2010.

[5] H. Godrich, A. M. Haimovich, and H. V. Poor, "Localization performance of coherent MIMO radar systems subject to phase synchronization errors," in 4th International Symposium on Communications, Control and Signal Processing (ISCCSP), Mar. 2010.

[6] M. Akçakaya and A. Nehorai, "MIMO radar detection and adaptive design under a phase synchronization mismatch," IEEE Transactions on Signal Processing, no. 10, pp. 4994 5005, Oct. 2010.

[7] Y. Rockah and P.M.Schultheiss, "Array shape calibration using sources in unknown locations-part i: Far-field sources," IEEE Transactions on Acoustics, Speech, and Signal Processing, no. 3, pp. 286-299, Mar. 1987.

[8] M. Viberg and A. L. Swindlehurst, "Analysis of the combined effects of finite samples and model errors on array processing performance," IEEE Transactions on Signal Processing, no. 11, pp. 3073-3083, Nov. 1994.

[9] A. Ferréol, P. Larzabal, and M. Viberg, "Statistical analysis of the MUSIC algorithm in the presence of modeling errors, takng into account the resolution probability," IEEE Transactions on Signal Processing, no. 58, pp. 4156-4166, Aug. 2010.

[10] S. M. Kay, Fundamentals of Statistical Signal Processing: Estimation Theory. Upper Saddle River, NJ, USA: PrenticeHall, Inc., Mar. 1993, vol. 1.

[11] A. Renaux, P. Forster, E. Chaumette, and P. Larzabal, "On the high-SNR conditional maximum-likelihood estimator full statistical characterization," IEEE Transactions on Signal Processing, vol. 54, no. 12, pp. 4840-4843, Dec. 2006. 